\title{
Two Fluid Shear-Free Composites
}

\author{
J.P. Krisch and E.N. Glass \\ Department of Physics, University of Michigan, Ann Arbor, MI
}

(Dated: 28 July 2013)

\begin{abstract}
Shear-free composite fluids are constructed from two Letelier rotated unaligned perfect fluids. The component fluid parameters necessary to construct a shear-free composite are investigated. A metric in the Stephani-Barnes solution family and a simple stationary metric are discussed.

PACS numbers: 04.40.Dg
\end{abstract}

\section{INTRODUCTION}

Complex fluids with properties such as anisotropy and heat flow, can often be modeled by several component perfect fluids in relative motion [1-4]. The components can be real separate fluids with different properties or, as Andersson and Comer [5] have suggested, could separately model the properties of a single fluid. Multi-fluid models are used to describe small astrophysical systems [6-10] as well as systems, like our Universe, that contain multiple matter content. That content appears in combinations of radiation, particle matter, dark energy, dark matter, and other more exotic materials, [11-16] and is also useful in relativistic fluid dynamics [17-22]. While three and higher [23-26] composite fluid models have been suggested, the simplest anisotropic composite stress-energy is written as a sum of two perfect fluids. Letelier [1] suggested a way of combining two perfect fluids that provides an anisotropic fluid with no heat flow. His model is enforced by an assumed relation between the component equations of state. The method will accommodate heat flow without this assumption [17], but there is an equation of state for the composite fluid that represents a generalization of the usual pressure isotropy condition [27-29]. How the fluid parameters of the composite are driven by the parameters of the component fluids is important, given the recent interest in shear-free perfect fluids [30] and the shear-free fluid conjecture [31 35]. With the increasing use of multi-fluid models, understanding the effects caused by component fluid motions can provide insights to the origin of composite fluid velocity components such 
as shear, expansion, and vorticity.

In this paper we construct shear-free composites from perfect fluids and examine how the composite fluid stress-energy and metric depend on the component fluid alignment. The component fluids are combined using the Letelier method with an alignment condition and this is briefly reviewed in the next section. The component/composite fluid parameters and stress-energy are connected to the metric for the containing spacetime. In the third section, we consider two metric examples. The first metric is taken from the Stephani-Barnes solution family [36] and uses two shear-free components. The second example describes a simple rotating spacetime and its concomitant vorticity. Three Appendices relating the composite/component fluid properties are provided.

\section{TWO FLUID FORMALISM WITH ALIGNMENT}

\section{Transformations and Composite Stress-Energy}

Consider a manifold which contains two perfect fluids and metric $g_{a b}$. A composite stressenergy for the perfect fluids is

$$
T_{a b}=\left(\varepsilon_{1}+p_{1}\right) U_{a}^{(1)} U_{b}^{(1)}+\left(\varepsilon_{2}+p_{2}\right) U_{a}^{(2)} U_{b}^{(2)}+\left(p_{1}+p_{2}\right) g_{a b}
$$

A single non-perfect fluid stress-energy tensor can be constructed by transforming the two timelike component fluid velocities $\left[U_{i}^{(1)}, U_{i}^{(2)}\right]$ into an unnormed timelike, spacelike pair $\left[U_{i}^{*}, \Upsilon_{i}^{*}\right]$ related to the unit pair, $\left[\hat{U}^{i}, \hat{\Upsilon}^{i}\right]$ by $N_{u} \hat{U}^{i}=U^{i *}$ and $N_{\Upsilon} \hat{\Upsilon}^{i}=\Upsilon^{i *}$. The composite fluid will be described by a tetrad $\left[U^{a}, \Upsilon^{a}, X^{a}, Z^{a}\right]$, where $X^{a}$ and $Z^{a}$ are any two spatial vectors orthogonal to $\Upsilon^{a}$. The basic velocity transformations with inverse are

$$
\begin{gathered}
{\left[\begin{array}{l}
N_{u} \hat{U}_{a} \\
N_{\Upsilon} \hat{\Upsilon}_{a}
\end{array}\right]=\left[\begin{array}{cc}
\cos \alpha & B \sin \alpha \\
-B^{-1} \sin \alpha & \cos \alpha
\end{array}\right]\left[\begin{array}{l}
U_{a}^{(1)} \\
U_{a}^{(2)}
\end{array}\right]} \\
{\left[\begin{array}{l}
U_{a}^{(1)} \\
U_{a}^{(2)}
\end{array}\right]=\left[\begin{array}{cc}
\cos \alpha & -B \sin \alpha \\
B^{-1} \sin \alpha & \cos \alpha
\end{array}\right]\left[\begin{array}{l}
N_{u} \hat{U}_{a} \\
N_{\Upsilon} \hat{\Upsilon}_{a}
\end{array}\right]}
\end{gathered}
$$

The normalization factors are

$$
\begin{aligned}
& N_{\Upsilon}^{2}=\Upsilon^{a *} \Upsilon_{a}^{*}=B^{-2}\left(\sin ^{2} \alpha-B^{2} \cos ^{2} \alpha\right) / \cos 2 \alpha \\
& N_{u}^{2}=-U^{* a} U_{a}^{*}=\left(\cos ^{2} \alpha-B^{2} \sin ^{2} \alpha\right) / \cos 2 \alpha .
\end{aligned}
$$


The general rotation angle, $\alpha$, is fixed with terms of the component velocity overlap by requiring $\Upsilon^{a} U_{a}=0, \Upsilon^{a}$ spacelike and $U^{a}$ timelike.

$$
\digamma=U^{(1) a} U_{a}^{(2)}=-\frac{1-B^{2}}{2 B} \tan 2 \alpha
$$

The overlap, $U^{(1) a} U_{a}^{(2)}$, between the component four-velocities is assumed constant. Substituting $U_{a}^{(1)}$ and $U_{a}^{(2)}$ from Eq.(3) into stress-energy Eq.(1), the composite stress-energy is

$$
T_{a b}=(\varepsilon+\Pi) \hat{U}_{a} \hat{U}_{b}+(P-\Pi) \hat{\Upsilon}_{a} \hat{\Upsilon}_{b}+\Pi g_{a b}+Q_{a} \hat{U}_{b}+Q_{b} \hat{U}_{a}
$$

with components

$$
\begin{aligned}
\Pi & =p_{1}+p_{2} \\
\varepsilon+\Pi & =N_{u}^{2}\left[\left(\varepsilon_{1}+p_{1}\right) \cos ^{2} \alpha+B^{-2}\left(\varepsilon_{2}+p_{2}\right) \sin ^{2} \alpha\right] \\
P-\Pi & =N_{\Upsilon}^{2}\left[B^{2}\left(\varepsilon_{1}+p_{1}\right) \sin ^{2} \alpha+\left(\varepsilon_{2}+p_{2}\right) \cos ^{2} \alpha\right] \\
Q_{a} & =Q \hat{\Upsilon}_{a}, \quad Q=N_{u} N_{\Upsilon}\left[B^{-1}\left(\varepsilon_{2}+p_{2}\right)-B\left(\varepsilon_{1}+p_{1}\right)\right] \sin \alpha \cos \alpha
\end{aligned}
$$

The anisotropic stress is $P$, and $Q_{a}$ is the heat current associated with $\hat{\Upsilon}_{a}$. Letelier [1]

defined $B=\sqrt{\frac{\varepsilon_{2}+p_{2}}{\varepsilon_{1}+p_{1}}}$ in order to zero the off diagonal stress-energy heat flow term, leaving a 1-parameter description of the composite fluid. A convenient way to define $B$ that maintains the heat flow is $B=\tan \alpha_{0}[17]$. With this choice of $B$, the velocity overlap equation, Eq.(15) can be rewritten.

$$
\digamma=U^{(1) a} U_{a}^{(2)}=-\frac{\tan 2 \alpha}{\tan 2 \alpha_{0}}
$$

The fluids are aligned when $\alpha=\alpha_{0}$. To maintain the spacelike/timelike nature of $\Upsilon^{a *}$ and $U^{a *}$, the range for $\left(\alpha, \alpha_{0}\right)$ is $0<\left(\alpha, \alpha_{0}\right)<\pi / 4$. For $\alpha=\alpha_{0}$, with two aligned components, the equation of state simply gives the isotropic stress, $P=\Pi$. For aligned fluids $N_{\Upsilon}=0$; the heat flow vanishes and the composite is another isotropic perfect fluid.

\section{Composite Equation of State}

The alignment restriction of $B=\tan \alpha_{0}$ removes the equation of state assumption on the component fluids, allows non-zero heat flow, and links the fluid description to the alignment of the component velocities. The definition of $B=\tan \alpha_{0}$ does impose an equation of state 
on the system. Unlike the Letelier assumption, the imposed equation of state involves the composite fluid parameters and follows from the definitions of those parameters.

$$
(\varepsilon+\Pi) S^{2}\left(\alpha, \alpha_{0}\right)+(\Pi-P) C^{2}\left(\alpha, \alpha_{0}\right)+2 Q \cot (2 \alpha) S\left(\alpha, \alpha_{0}\right) C\left(\alpha, \alpha_{0}\right)=0
$$

Eq.(9) is an identity in term of the component parameters and imposes no component conditions. It can be used as a differential equation for the metric functions or replace the field equation for the heat scalar. The two functions, $C^{2}\left(\alpha, \alpha_{0}\right)$ and $S^{2}\left(\alpha, \alpha_{0}\right)$ are related to the normalization constants

$$
\begin{aligned}
& C^{2}\left(\alpha, \alpha_{0}\right)=N_{u}^{2} \cos (2 \alpha) \cos ^{2}\left(\alpha_{0}\right) \\
& S^{2}\left(\alpha, \alpha_{0}\right)=N_{\Upsilon}^{2} \cos (2 \alpha) \sin ^{2}\left(\alpha_{0}\right)
\end{aligned}
$$

For $\alpha=\alpha_{0}$, the equation of state gives the isotropic stress result, $P=\Pi$. The size of the stress anisotropy rises as the fluids become more and more unaligned. When the field equations are substituted for the fluid parameters, this equation of state provides a generalization of the metric condition imposed by stress isotropy [27-29]. The stress-energy following from the field equations is implicit in the equation of state, but it can be useful in providing an alignment related differential equation for the metric potentials, or used to replace a field equation. In the next section we give two examples.

\section{METRIC EXAMPLES}

\section{Stephani-Barnes metric}

In this section, we consider the spherically symmetric metric in the Stephani-Barnes [36, 37] family and examine the properties of the composite fluid that could result from two shear-free perfect fluid components with a velocity in the radial direction.

$$
d s^{2}=L(t, r)^{-2}\left[-N(t, r)^{2} d t^{2}+d r^{2}+r^{2} d \Omega^{2}\right]
$$

The single composite fluid has a co-moving four velocity

$$
U^{a}=\left[\frac{L(t, r)}{N(t, r)}, 0,0,0\right]
$$


The composite velocity is shear-free with expansion $\theta=-3 \dot{L} / N$. From Eq.(3), the component four velocities that can build the composite are written as

$$
U^{(i) a}=A^{(i)} \hat{U}^{a}+C^{(i)} \hat{\Upsilon}^{a}
$$

with

$$
\begin{aligned}
& A^{(1)}=\cos \alpha N_{u}, \quad C^{(1)}=-B \sin \alpha N_{\Upsilon} \\
& A^{(2)}=B^{-1} \sin \alpha N_{u}, \quad C^{(2)}=\cos \alpha N_{\Upsilon}
\end{aligned}
$$

While the composite is shear-free, the component velocities, with spatial components, in general have shear. Choosing $\Upsilon^{a}$ in the radial direction, the component fluid velocities are

$$
U_{a}^{(i)}=\left[-\frac{A^{(i)} N}{L}, \frac{C^{(i)}}{L}, 0,0\right] .
$$

For this velocity the shear tensor components of the component fluids all depend on the same term

$$
\sigma_{a b} \sim\left(\frac{N_{, r}}{N}-\frac{1}{r}\right)
$$

For the components to be shear-free, $N(t, r)$ must be separable, with form

$$
N(t, r)=r N(t)
$$

Absorbing the factor $N(t)$ into the time coordinate, the metric that could contain shear-free components with radial spatial velocities is

$$
d s^{2}=L(t, r)^{-2}\left[-r^{2} d t^{2}+d r^{2}+r^{2} d \Omega^{2}\right] .
$$

This metric is a special case of a metric considered by Sussman and reviewed by Krasinski [36, 38]. The metric form in Eq. (17) has been discussed in the literature under a number of special conditions $[27-29,37]$. 


\section{Stress Energy}

The field equations provide the composite stress-energy components

$$
\begin{aligned}
8 \pi \varepsilon & =\frac{3\left(L_{, t}\right)^{2}}{r^{2}}+L^{2}\left[2 \frac{L, r r}{L}-3\left(\frac{L,_{r}}{L}\right)^{2}+\frac{4}{r}\left(\frac{L, r}{L}\right)\right] \\
8 \pi \Pi & =\frac{L^{2}}{r^{2}}\left[2 \frac{L, t t}{L}-3\left(\frac{L, t}{L}\right)^{2}\right]+L^{2}\left[-2 \frac{L, r r}{L}+3\left(\frac{L,_{r}}{L}\right)^{2}-\frac{4}{r}\left(\frac{L,_{r}}{L}\right)+\frac{1}{r^{2}}\right] \\
8 \pi P_{r} & =\frac{L^{2}}{r^{2}}\left[2 \frac{L, t t}{L}-3\left(\frac{L, t}{L}\right)^{2}\right]+L^{2}\left[3\left(\frac{L, r}{L}\right)^{2}-\frac{6}{r}\left(\frac{L,_{r}}{L}\right)+\frac{2}{r^{2}}\right] \\
Q_{r} & =-2\left(\frac{L, t}{r}\right)_{, r}
\end{aligned}
$$

Substituting the fluid parameters into the equation of state from the Letelier composition Eq.(9), one finds

$$
\frac{2 L, t t}{L}-\frac{4 r^{2}}{L} \partial_{r}\left(\frac{L, t}{r}\right) \cot (2 \alpha) \frac{C\left(\alpha, \alpha_{0}\right)}{S\left(\alpha, \alpha_{0}\right)}+2 r^{2}\left(\frac{L,_{r}}{r L}-\frac{L,_{r r}}{L}-\frac{1}{2 r^{2}}\right) \frac{C^{2}\left(\alpha, \alpha_{0}\right)}{S^{2}\left(\alpha, \alpha_{0}\right)}+1=0 .
$$

If, for example, $L=L(r)$ the equation of state is

$$
\frac{L_{, r r}}{L}-\frac{L_{, r}}{r L}+\frac{1}{2 r^{2}}=0 .
$$

an Euler equation, homogeneous in $r$ of degree -2. The stresses are isotropic. The additional structure in equation (19) extends the single fluid isotropy condition. The case where $L$ is only a function of time, $L=L(t)$, is not isotropic. For this case, the equation of state becomes a simple differential equation for $L(t)$.

$$
\frac{2 L, t t}{L}+\frac{4 L, t}{L} \cot (2 \alpha) \frac{C\left(\alpha, \alpha_{0}\right)}{S\left(\alpha, \alpha_{0}\right)}-\frac{C^{2}\left(\alpha, \alpha_{0}\right)}{S^{2}\left(\alpha, \alpha_{0}\right)}+1=0 .
$$

For unaligned fluids the metric function $L(t)$ is an exponential sum depending on the alignment of the two perfect fluids. A simple exponential solution is

$$
L(t)=L_{1} e^{\beta t}
$$

$\beta$ is related to $\left(\alpha, \alpha_{0}\right)$ through the equation of state and satisfies the quadratic equation

$$
\beta^{2}+2 \beta\left[\cot (2 \alpha) \frac{C\left(\alpha, \alpha_{0}\right)}{S\left(\alpha, \alpha_{0}\right)}\right]+\left[\frac{S^{2}\left(\alpha, \alpha_{0}\right)-C^{2}\left(\alpha, \alpha_{0}\right)}{2 S^{2}\left(\alpha, \alpha_{0}\right)}\right]=0
$$


The fluid content and curvature scalar are

$$
\begin{aligned}
8 \pi \varepsilon & =\frac{L^{2}}{r^{2}}\left(3 \beta^{2}\right) \\
8 \pi \Pi & =\frac{L^{2}}{r^{2}}\left(1-\beta^{2}\right) \\
8 \pi P_{r} & =\frac{L^{2}}{r^{2}}\left(2-\beta^{2}\right) \\
Q & =\frac{L^{2}}{r^{2}}(2 \beta) \\
R & =\frac{L^{2}}{r^{2}} 2\left(3 \beta^{2}-2\right)
\end{aligned}
$$

Positive stress requires $0<\beta^{2}<1$. This solution does not have an isotropic limit and exhibits a curvature dependence on alignment.

\section{The component fluids}

The shear-free velocity components for this example are

$$
U_{a}^{(i)}=\left[-\frac{A^{(i)} r}{L}, \frac{C^{(i)}}{L}, 0,0\right] .
$$

with component expansions

$$
\theta_{(i)}=3 \frac{-A_{(i)} L,{ }_{t}+C_{(i)} L}{r}
$$

In Appendix A, we examine the conditions necessary for two shear-free components to combine in a shear-free composite. One of the conditions relates the composite and component expansions

$$
N_{u} \theta=-3 N_{u} L, t / r=\cos \alpha \theta_{1}+B \sin \alpha \theta_{2}
$$

This is satisfied for these shear-free components, with the $C^{(i)}$ component contributions cancelling. An interesting insight is that the component fluids can be expanding or contracting due to their $C^{(i)}$ contribution, while the sign of the composite expansion is determined by the metric. A second condition is that the two component fluids must have unequal accelerations along the direction of anisotropy. For the components in this example, the 
accelerations are

$$
\begin{aligned}
\dot{U}_{a}^{(i)} & =\left[C^{(i)} \frac{L, t}{L}-A^{(i)}\right]\left[C^{(i)},-\frac{A^{(i)}}{r}, 0,0\right] \\
C^{(1)} \frac{L, t}{L}-A^{(1)} & =B N_{\Upsilon}\left(-\sin \alpha \frac{L, t}{L}-\frac{N_{u}}{B N_{\Upsilon}} \cos \alpha\right) \\
C^{(2)} \frac{L, t}{L}-A^{(2)} & =N_{\Upsilon}\left(\cos \alpha \frac{L, t}{L}-\frac{N_{u}}{B N_{\Upsilon}} \sin \alpha\right)
\end{aligned}
$$

with clearly unequal acceleration. One of the components could be unaccelerated. For example, if fluid 2 is unaccelerated

$$
\frac{L, t}{L}=\frac{A^{(2)}}{C^{(2)}}=\frac{N_{u}}{B N_{\Upsilon}} \tan \alpha
$$

then the second fluid also has zero expansion and the metric is determined by the alignment conditions.

\section{A Simple Rotating Metric}

The second metric example is a simple cylindrical stationary spacetime with metric

$$
d s^{2}=-d t^{2}-2 f(r) d \phi d t+r^{2} d \phi^{2}+d r^{2}+d z^{2}
$$

The composite fluid will be constructed from two perfect fluids with shear and vorticity. The motion and stress energy will be decomposed using a comoving tetrad.

$$
U_{a}=-[1,0, f, 0], \quad R_{a}=[0,1,0,0], \quad \Phi_{a}=[0,0, D, 0], \quad Z_{a}=[0,0,0,1] .
$$

The stress-energy of the composite fluid is, with $D^{2}=r^{2}+f^{2}$

$$
\begin{aligned}
8 \pi \varepsilon & =-\frac{D, r r}{D}+3 \frac{f,_{r}^{2}}{D^{2}} \\
8 \pi P_{\phi} & =\frac{f,_{r}^{2}}{D^{2}} \\
8 \pi P_{r} & =\frac{f,_{r}^{2}}{D^{2}} \\
8 \pi P_{z} & =\frac{D, r r}{D}-\frac{f,_{r}}{D^{2}}
\end{aligned}
$$

Since the alignment formalism describes a fluid with a single anisotropic stress, the direction of anisotropy is $Z^{i}$ and there will be heat flow down the axis of the cylindrical system.

$$
Q \cot (2 \alpha) S\left(\alpha, \alpha_{0}\right) C\left(\alpha, \alpha_{0}\right)=\frac{D,_{r r}}{2 D}\left[S^{2}\left(\alpha, \alpha_{0}\right)+C^{2}\left(\alpha, \alpha_{0}\right)\right]-\frac{f_{r_{r}}^{2}}{D^{2}}\left[2 S^{2}\left(\alpha, \alpha_{0}\right)+C^{2}\left(\alpha, \alpha_{0}\right)\right]
$$


The velocity of the composite is shear and expansion free, with vorticity $\omega_{r \phi}=f_{, r} / 2$. The component velocities have a component in the z-direction.

$$
U^{(i) a}=A^{(i)} \hat{U}^{a}+C^{(i)} Z^{a} .
$$

and can have shear and vorticity. The standard vorticity definition based on the $U_{a}^{(i)}$ covariant derivative expansion is

$$
\begin{aligned}
\omega_{a b}^{(i)} & =U_{[a ; b]}^{(i)}+\dot{U}_{[a}^{(i)} U_{b]}^{(i)} \\
U^{(i) a} \omega_{a b}^{(i)} & =0
\end{aligned}
$$

The component vorticities could have a time like component with the velocity constraint providing the relation

$$
\left[A^{(i)}-\frac{C^{(i)} f}{D}\right] \omega_{t b}^{(i)}=-\frac{C^{(i)}}{D} \omega_{\phi b}^{(i)}
$$

If the fluids are aligned, $C^{(i)}=0\left(N_{\Upsilon}=0\right)$, there is only the single composite vorticity, $\omega_{r \phi}$,as the components rotate with the composite value.

This description could be compared to the two-fluid model based on momentum [39, 40]. In these models the fluid current, $n^{(i) a}$, and momentum $\mu^{(i) a}$, are defined in terms of the component velocities and currents

$$
\begin{aligned}
n^{(1) a} & =n^{(1)} U^{(1) a} \\
\mu_{a}^{(1)} & =B_{(1)} n^{(1)} U_{a}^{(1)}+A_{(12)} n^{(2)} U_{a}^{(2)}
\end{aligned}
$$

with $B_{(1)}$ and $A_{(12)}$ constants of the formalism. The vorticity is based on the momentum and, for $i=1$ (fluid $\mathrm{x}$ in their notation) is defined as

$$
\begin{aligned}
\bar{\omega}_{a b}^{(1)} & =\mu_{a ; b}^{(1)}-\mu_{b ; a}^{(1)} \\
U^{(1) a} \bar{\omega}_{a b}^{(1)} & =0
\end{aligned}
$$

For the simple stationary metric of this example with only r-dependence, the momentum based vorticities are

$$
\begin{aligned}
\bar{\omega}_{t b}^{(1)} & =\partial_{b} \mu_{t}^{(1)} \\
\bar{\omega}_{\phi b}^{(1)} & =\partial_{b} \mu_{\phi}^{(1)} \\
U^{(1) t} \partial_{b} \mu_{t}^{(1)} & =-U^{(1) \phi} \partial_{b} \mu_{\phi}^{(1)}
\end{aligned}
$$


If the velocities are aligned and taken comoving, conditions are placed on the functional structure of the component momentum. The use of a velocity constraint on a momentum based vorticity also raises questions about fluid motion in more complicated metrics. Spin fluid calculations have demonstrated different physical descriptions arising from imposing a momentum versus velocity constraint [41 43] on a spin tensor.

\section{DISCUSSION}

In summary, we have considered a shear-free anisotropic composite fluid constructed from two perfect fluids and shown that, when the component fluid alignment is considered, a composite equation of state is imposed which extends the usual isotropy condition. We considered two examples. The first used two shear-free component fluids with a radial velocity component. The metric for the fluid provided a specific solution to a family of conformal Killing vector metrics discussed by Sussman [38]. The second example considered a simple rotating fluid. This example illustrated a possible difference in the alignment description used in this paper and the momentum/current description.

We have focused on the composite fluid produced by combining two perfect fluids. However, the inverse Letelier expansion of two perfect fluids, in terms of the composite four velocity and anisotropy vector, can be related to a potential representation. For example, Schutz [44], expanding the minimal set of Clebsch potentials, introduced a six-potential representation for a perfect fluid four velocity.

$$
U_{a}=\mu^{-1}\left(\varphi,{ }_{a}+\alpha_{c} \beta_{c}, a+C s_{, a}\right)
$$

$\mu^{-1}$ is the specific inertial mass. $s$ is the specific entropy. $C$ (usually called $\theta$ ), is the thermasy of Van Danzig [45], $\alpha_{c}$ and $\beta_{c}$ are the Clebsch potentials [44] needed for a fluid with vorticity. In the Letelier expansion, each of the component velocities for the anisotropic fluid is expanded with a minimal vector set related to the component fluid alignment.

$$
U_{a}^{(i)}=A^{(i)} \hat{U}_{a}+C^{(i)} \hat{\Upsilon}_{a}
$$

Combining the Schutz potentials with a multifluid combination method could relate the alignment parameters to velocity potentials and provide a broader multifluid formalism. 


\section{Appendix A: Relating the component fluid accelerations}

The component velocity overlap equation enforces the orthogonality of the transformed $\left[U_{a}, \Upsilon_{a}\right]$ pair.

$$
\digamma:=U^{(1) a} U_{a}^{(2)}=-\frac{\left(1-B^{2}\right)}{2 B} \tan 2 \alpha
$$

$\alpha=\alpha_{0}$ is the condition for $U_{a}^{(1)}$ and $U_{a}^{(2)}$ to be aligned. $\alpha$ varies the size of the component spatial speeds. Assuming constant angles the covariant derivative is

$$
U_{a ; b}^{(1)} U^{(2) a}=-U^{(1) a} U_{a ; b}^{(2)}
$$

Expanding the derivatives and forming the products with $U^{(2) b}$ and, separately with $U^{(1) b}$, we have

$$
\begin{aligned}
{\left.\left[-\dot{U}_{a}^{(1)} U_{b}^{(1)}+\omega_{a b}^{(1)}+\sigma_{a b}^{(1)}+\frac{\theta_{(1)}}{3} h_{a b}^{(1)}\right)\right] U^{(2) a} } & =-\left[-\dot{U}_{a}^{(2)} U_{b}^{(2)}+\omega_{a b}^{(2)}+\sigma_{a b}^{(2)}+\frac{\theta_{(2)}}{3} h_{a b}^{(2)}\right] U^{(1) a} \quad(\mathrm{~A} 2) \\
\dot{U}_{a}^{(1)} U^{(2) a} & =\dot{U}_{a}^{(2)} U^{(1) a} \digamma-\sigma_{a b}^{(2)} U^{(1) b} U^{(1) a}-\frac{\theta_{(2)}}{3} h_{a b}^{(2)} U^{(1) b} U^{(1) a} \\
\dot{U}_{a}^{(2)} U^{(1) a} & =\dot{U}_{a}^{(1)} U^{(2) a} \digamma-\sigma_{a b}^{(1)} U^{(2) a} U^{(2) b}-\frac{\theta_{(1)}}{3} h_{a b}^{(1)} U^{(2) b} U^{(2) a}
\end{aligned}
$$

Substituting we find the two equations for the acceleration

$$
\begin{aligned}
& \dot{U}_{a}^{(1)} U^{(2) a}\left(1-\digamma^{2}\right)=\left(\digamma \frac{\theta_{(1)}}{3}+\frac{\theta_{(2)}}{3}\right)\left(1-\digamma^{2}\right)-\digamma \sigma_{a b}^{(1)} U^{(2) a} U^{(2) b}-\sigma_{a b}^{(2)} U^{(1) b} U^{(1) a} \\
& \dot{U}_{a}^{(2)} U^{(1) a}\left(1-\digamma^{2}\right)=\left(\frac{\theta_{(1)}}{3}+\digamma \frac{\theta_{(2)}}{3}\right)\left(1-\digamma^{2}\right)-\sigma_{a b}^{(1)} U^{(2) a} U^{(2) b}-\digamma \sigma_{a b}^{(2)} U^{(1) b} U^{(1) a}
\end{aligned}
$$

For shear-free components the expression simplifies to

$$
\begin{aligned}
\dot{U}_{a}^{(1)} U^{(2) a} & =\left(\digamma \frac{\theta_{(1)}}{3}+\frac{\theta_{(2)}}{3}\right) \\
\dot{U}_{a}^{(2)} U^{(1) a} & =\left(\frac{\theta_{(1)}}{3}+\digamma \frac{\theta_{(2)}}{3}\right)
\end{aligned}
$$

\section{Appendix B: Composite fluid parameters}

In this Appendix we use the Letelier transformation equations to develop expressions for the acceleration, expansion, shear and vorticity of the composite fluid. The transformation 
equations are

$$
\begin{aligned}
N_{u} U_{a} & =(\cos \alpha) U_{a}^{(1)}+(B \sin \alpha) U_{a}^{(2)} \\
N_{\Upsilon} \Upsilon_{a} & =-\left(B^{-1} \sin \alpha\right) U_{a}^{(1)}+(\cos \alpha) U_{a}^{(2)} \\
U_{a}^{(1)} & =\left(\cos \alpha N_{U}\right) U_{a}-\left(B \sin \alpha N_{\Upsilon}\right) \Upsilon_{a} \\
U_{a}^{(2)} & =\left(B^{-1} \sin \alpha N_{U}\right) U_{a}+\left(\cos \alpha N_{\Upsilon}\right) \Upsilon_{a}
\end{aligned}
$$

Taking the covariant derivative of $U_{a}$ and expanding one obtains

$$
\begin{aligned}
N_{u}\left(-\dot{U}_{a} U_{b}+\omega_{a b}+\sigma_{a b}+\frac{\theta}{3} h_{a b}\right) & =\cos \alpha\left(-\dot{U}_{a}^{(1)} U_{b}^{(1)}+\omega_{a b}^{(1)}+\sigma_{a b}^{(1)}+\frac{\theta_{(1)}}{3} h_{a b}^{(1)}\right) \\
& +B \sin \alpha\left(-\dot{U}_{a}^{(2)} U_{b}^{(2)}+\omega_{a b}^{(2)}+\sigma_{a b}^{(2)}+\frac{\theta_{(2)}}{3} h_{a b}^{(2)}\right)
\end{aligned}
$$

\section{Composite Expansion and Acceleration}

\section{Expansion}

Taking the trace of Eq.(B2), the composite and component expansions are related by

$$
N_{u} \theta=\cos \alpha \theta_{(1)}+B \sin \alpha \theta_{(2)}
$$

\section{Acceleration}

The acceleration follows from the covariant derivative and we have

$$
\begin{aligned}
N_{u} \dot{U}_{a} & =\cos \alpha\left(-\dot{U}_{a}^{(1)} U_{b}^{(1)} U^{b}+\sigma_{a b}^{(1)} U^{b}+\omega_{a b}^{(1)} U^{b}+\frac{\theta_{(1)}}{3} U^{b} h_{a b}^{(1)}\right) \\
& +B \sin \alpha\left(-\dot{U}_{a}^{(2)} U_{b}^{(2)} U^{b}+\sigma_{a b}^{(2)} U^{b}+\omega_{a b}^{(2)} U^{b}+\frac{\theta_{(2)}}{3} U^{b} h_{a b}^{(2)}\right)
\end{aligned}
$$

Using the transformation equations to generate the velocity dot products, the acceleration can be rewritten

$$
\begin{aligned}
\dot{U}_{a} & =\cos ^{2} \alpha \dot{U}_{a}^{(1)}+\frac{B \sin \alpha \cos \alpha}{N_{u}^{2}}\left[\sigma_{a b}^{(1)} U^{(2) b}+\omega_{a b}^{(1)} U^{(2) b}+\frac{\theta_{(1)}}{3}\left(U_{a}^{(2)}+\digamma U_{a}^{(1)}\right)\right] \\
& +\sin ^{2} \alpha \dot{U}_{a}^{(2)}+\frac{B \sin \alpha \cos \alpha}{N_{u}^{2}}\left[\sigma_{a b}^{(2)} U^{(1) b}+\omega_{a b}^{(2)} U^{(1) b}+\frac{\theta_{(2)}}{3}\left(U_{a}^{(1)}+\digamma U_{a}^{(2)}\right)\right]
\end{aligned}
$$




\section{Acceleration with Shear-Free components}

When the components are shear-free, the expression for the composite acceleration simplifies, with the vorticity as well as the shear terms vanishing. To see this, consider the symmetric and antisymmetric combinations of Eq.(B2):

$$
\begin{aligned}
N_{u}\left(-\dot{U}_{a} U_{b}-\dot{U}_{b} U_{a}+2 \sigma_{a b}+\frac{2 \theta}{3} h_{a b}\right)= & \cos \alpha\left[-\dot{U}_{a}^{(1)} U_{b}^{(1)}-\dot{U}_{b}^{(1)} U_{a}^{(1)}+2 \sigma_{a b}^{(1)}+\frac{2 \theta_{(1)}}{3} h_{a b}^{(1)}\right] \\
+ & B \sin \alpha\left[-\dot{U}_{a}^{(2)} U_{b}^{(2)}-\dot{U}_{b}^{(2)} U_{a}^{(2)}+2 \sigma_{a b}^{(2)}+\frac{2 \theta_{(2)}}{3} h_{a b}^{(2)}\right] \\
N_{u}\left(-\dot{U}_{a} U_{b}+\dot{U}_{b} U_{a}+2 \omega_{a b}\right) & =\cos \alpha\left[-\dot{U}_{a}^{(1)} U_{b}^{(1)}+\dot{U}_{b}^{(1)} U_{a}^{(1)}+2 \omega_{a b}^{(1)}\right] \\
& +B \sin \alpha\left[-\dot{U}_{a}^{(2)} U_{b}^{(2)}+\dot{U}_{b}^{(2)} U_{a}^{(2)}+2 \omega_{a b}^{(2)}\right]
\end{aligned}
$$

The acceleration can be obtained from either the symmetric or the antisymmetric equation. Using both will provide the vorticity relation. First using the symmetric combination, multiplying by $U^{b}$ we have

$$
\begin{aligned}
N_{u} \dot{U} & =\cos \alpha\left(-\dot{U}_{a}^{(1)} U_{b}^{(1)} U^{b}-\dot{U}_{b}^{(1)} U^{b} U_{a}^{(1)}+2 U^{b} \sigma_{a b}^{(1)}+\frac{2 \theta_{(1)}}{3} h_{a b}^{(1)} U^{b}\right) \\
& +B \sin \alpha\left(-\dot{U}_{a}^{(2)} U_{b}^{(2)} U^{b}-\dot{U}_{b}^{(2)} U^{b} U_{a}^{(2)}+2 U^{b} \sigma_{a b}^{(2)}+\frac{2 \theta_{(2)}}{3} U^{b} h_{a b}^{(2)}\right)
\end{aligned}
$$

Using the equation for $U^{b}$ in terms of $U^{b(1)}$ and $U^{b(2)}$, this can be rewritten.

$$
\begin{aligned}
\dot{U}_{a} & =\dot{U}_{a}^{(1)} \cos ^{2} \alpha+\dot{U}_{a}^{(2)} \sin ^{2} \alpha-\frac{B \sin \cos \alpha}{N_{u}^{2}}\left[\dot{U}_{b}^{(1)} U^{b(2)} U_{a}^{(1)}+\dot{U}_{b}^{(2)} U^{b(1)} U_{a}^{(2)}\right] \\
& +\frac{2 B \sin \alpha \cos \alpha}{N_{u}^{2}}\left(U^{b(1)} \sigma_{a b}^{(2)}+U^{b(2)} \sigma_{a b}^{(1)}\right) \\
& +\frac{B \sin \cos \alpha}{N_{u}^{2}}\left[\frac{2 \theta_{(1)}}{3}\left(U_{a}^{(2)}+\digamma U_{a}^{(1)}\right)+\frac{2 \theta_{(2)}}{3}\left(U_{a}^{(1)}+\digamma U_{a}^{(2)}\right)\right]
\end{aligned}
$$

Using the same procedure with the anti-symmetric combination the acceleration involving the component vorticity is found.

$$
\begin{aligned}
\dot{U}_{a} & =\dot{U}_{a}^{(1)} \cos ^{2} \alpha+\dot{U}_{a}^{(2)} \sin ^{2} \alpha \\
& +\frac{B \sin \alpha \cos \alpha}{N_{u}^{2}}\left[\dot{U}_{b}^{(1)} U_{a}^{(1)} U^{b(2)}+\dot{U}_{b}^{(2)} U^{b(1)} U_{a}^{(2)}\right] \\
& +\frac{2 B \cos \alpha \sin }{N_{u}^{2}}\left(\omega_{a b}^{(1)} U^{b(2)}+U^{b(1)} \omega_{a b}^{(2)}\right)
\end{aligned}
$$


For the two results to be equal implies

$$
\begin{aligned}
& U^{(1) b} \sigma_{a b}^{(2)}+U^{(2) b} \sigma_{a b}^{(1)}-U^{(2) b} \omega_{a b}^{(1)}-U^{(1) b} \omega_{a b}^{(2)} \\
& =\dot{U}_{b}^{(1)} U_{a}^{(1)} U^{(2) b}+\dot{U}_{b}^{(2)} U^{(1) b} U_{a}^{(2)}-\frac{\theta_{(1)}}{3}\left(U_{a}^{(2)}+\digamma U_{a}^{(1)}\right)-\frac{\theta_{(2)}}{3}\left(U_{a}^{(1)}+\digamma U_{a}^{(2)}\right)
\end{aligned}
$$

Substituting for the acceleration velocity product from Eqs. (A3) A4 and combining, the component expansion contributions cancel and we have

$$
\begin{aligned}
& \left(\omega_{a b}^{(1)}-\sigma_{a b}^{(1)}\right) U^{b(2)}+U^{b(1)}\left(\omega_{a b}^{(2)}-\sigma_{a b}^{(2)}\right) \\
& =\frac{U_{a}^{(1)}\left(\sigma_{a b}^{(1)} U^{a(2)} U^{b(2)} \digamma+\sigma_{a b}^{(2)} U^{a(1)} U^{b(1)}\right)+U_{a}^{(2)}\left(\sigma_{a b}^{(2)} U^{a(1)} U^{b(1)} \digamma+\sigma_{a b}^{(1)} U^{a(2)} U^{b(2)}\right)}{\left(1-\digamma^{2}\right)}
\end{aligned}
$$

If the component shears are zero, this provides a relation between the component vorticities

$$
\omega_{a b}^{(1)} U^{(2) b}=-U^{(1) b} \omega_{a b}^{(2)}
$$

Substituting for the acceleration/velocity overlaps, the general composite acceleration for shear-free components is

$$
\dot{U}_{a}=\dot{U}_{a}^{(1)} \cos ^{2} \alpha+\dot{U}_{a}^{(2)} \sin ^{2} \alpha+\frac{B \sin \alpha \cos \alpha}{N_{u}^{2}}\left[\frac{\theta_{(1)} \digamma+\theta_{(2)}}{3} U_{a}^{(1)}+\frac{\theta_{(2)} \digamma+\theta_{(1)}}{3} U_{a}^{(2)}\right]
$$

The overlap of the composite acceleration onto the anisotropy vector for shear-free components is

$$
\dot{U}_{a} \Upsilon^{a}=\frac{-\theta_{(1)} \sin \alpha+\theta_{(2)} B \cos \alpha}{3 B N_{\Upsilon}}
$$

For expansion-free component fluids, this requires the acceleration and the direction of anisotropy to be orthogonal.

\section{Fluid Shear}

The composite fluid shear is the most important of the composite parameters as its form will set the component conditions for a shear-free composite. The fluid shear follows from Eq.(B2). The composite fluid tetrad is $\left[U^{a}, \Upsilon^{a}, X^{a}, Z^{a}\right]$, where $\Upsilon^{a}$ is the transformation anisotropy vector and $X^{a}$ and $Z^{a}$ are any other spatial vectors orthogonal to $\Upsilon^{a}$ and $U^{a}$. Any component velocity product with $X^{a}$ or $Z^{a}$ is zero since the component velocities are rotated into the pair $\left[U^{a}, \Upsilon^{a}\right]$. Using the symmetric combination, Eq.(B6), first multiply by 
$X^{a}$

$$
\begin{aligned}
N_{u}\left(-\dot{U}_{a} X^{a} U_{b}+2 X^{a} \sigma_{a b}+\frac{2 \theta}{3} X_{b}\right) & =\cos \alpha\left(-\dot{U}_{a}^{(1)} X^{a} U_{b}^{(1)}+2 X^{a} \sigma_{a b}^{(1)}+\frac{2 \theta_{(1)}}{3} X_{b}\right) \\
& +B \sin \alpha\left(-\dot{U}_{a}^{(2)} X^{a} U_{b}^{(2)}+2 X^{a} \sigma_{a b}^{(2)}+\frac{2 \theta_{(2)}}{3} X_{b}\right)
\end{aligned}
$$

The composite expansion is related to the component expansions, Eq.(B3). Using this we have

$$
N_{u}\left(-\dot{U}_{a} X^{a} U_{b}+2 X^{a} \sigma_{a b}\right)=\cos \alpha\left(-\dot{U}_{a}^{(1)} X^{a} U_{b}^{(1)}+2 X^{a} \sigma_{a b}^{(1)}\right)+B \sin \alpha\left(-\dot{U}_{a}^{(2)} X^{a} U_{b}^{(2)}+2 X^{a} \sigma_{a b}^{(2)}\right)
$$

Multiplying by the unit vectors $X^{b}$ and $Z^{b}$

$$
\begin{aligned}
& N_{u} X^{a} X^{b} \sigma_{a b}=X^{a} X^{b}\left(\cos \alpha \sigma_{a b}^{(1)}+B \sin \alpha \sigma_{a b}^{(2)}\right) \\
& N_{u} X^{a} Z^{b} \sigma_{a b}=X^{a} Z^{b}\left(\cos \alpha \sigma_{a b}^{(1)}+B \sin \alpha \sigma_{a b}^{(2)}\right)
\end{aligned}
$$

The shear component in the $Z^{a} Z^{a}$ direction follows directly by analogy

$$
N_{u} Z^{a} Z^{b} \sigma_{a b}=Z^{a} Z^{b}\left(\cos \alpha \sigma_{a b}^{(1)}+B \sin \alpha \sigma_{a b}^{(2)}\right)
$$

For shear-free components, these composite shear components will be zero. First repeating with a $\Upsilon^{b}$ contraction

$$
\begin{aligned}
N_{u}\left(-\dot{U}_{b} \Upsilon^{b} U_{a}+2 \Upsilon^{b} \sigma_{a b}+\frac{2 \theta}{3} \Upsilon_{a}\right) & =\cos \alpha\left[-\dot{U}_{a}^{(1)} \Upsilon^{b} U_{b}^{(1)}-\dot{U}_{b}^{(1)} \Upsilon^{b} U_{a}^{(1)}+2 \Upsilon^{b} \sigma_{a b}^{(1)}+\frac{2 \theta_{(1)}}{3} \Upsilon^{b} h_{a b}^{(1)}\right] \\
& +B \sin \alpha\left[-\dot{U}_{a}^{(2)} \Upsilon^{b} U_{b}^{(2)}-\dot{U}_{b}^{(2)} \Upsilon^{b} U_{a}^{(2)}+2 \Upsilon^{b} \sigma_{a b}^{(2)}+\frac{2 \theta_{(2)}}{3} \Upsilon^{b} h_{a b}^{(2)}\right]
\end{aligned}
$$

and then $X^{b}$ and $Z^{b}$

$$
\begin{aligned}
X^{b} \Upsilon^{a} \sigma_{a b} & =\frac{B \sin \alpha \cos \alpha N_{\Upsilon}}{2 N_{u}} X^{b}\left(\dot{U}_{b}^{(1)}-\dot{U}_{b}^{(2)}\right)+\frac{X^{b} \Upsilon^{a}}{N_{u}}\left(\cos \alpha \sigma_{a b}^{(1)}+B \sin \alpha \sigma_{a b}^{(2)}\right) \\
Z^{b} \Upsilon^{a} \sigma_{a b} & =\frac{B \sin \alpha \cos \alpha N_{\Upsilon}}{2 N_{u}} Z^{b}\left(\dot{U}_{b}^{(1)}-\dot{U}_{b}^{(2)}\right)+\frac{Z^{b} \Upsilon^{a}}{N_{u}}\left(\cos \alpha \sigma_{a b}^{(1)}+B \sin \alpha \sigma_{a b}^{(2)}\right)
\end{aligned}
$$

The last shear component $\Upsilon^{a} \Upsilon^{b} \sigma_{a b}$ can be calculated and it is where the shear-free conditions enter

$$
\begin{aligned}
N_{u}\left(2 \Upsilon^{a} \Upsilon^{b} \sigma_{a b}+\frac{2 \theta}{3}\right) & =\cos \alpha\left[\left(-\dot{U}_{a}^{(1)} \Upsilon^{a} \Upsilon^{b} U_{b}^{(1)}-\dot{U}_{b}^{(1)} \Upsilon^{a} \Upsilon^{b} U_{a}^{(1)}\right.\right. \\
& \left.+2 \Upsilon^{a} \Upsilon^{b} \sigma_{a b}^{(1)}+\frac{2 \theta_{(1)}}{3}\left(1+\Upsilon^{b} U_{b}^{(1)} \Upsilon^{a} U_{a}^{(1)}\right)\right] \\
& +B \sin \alpha\left[\left(-\dot{U}_{a}^{(2)} \Upsilon^{a} \Upsilon^{b} U_{b}^{(2)}-\Upsilon^{b} \dot{U}_{b}^{(2)} U_{a}^{(2)} \Upsilon^{a}+2 \Upsilon^{a} \Upsilon^{b} \sigma_{a b}^{(2)}\right.\right. \\
& \left.+\frac{2 \theta_{(2)}}{3}\left(1+\Upsilon^{b} U_{b}^{(2)} \Upsilon^{a} U_{a}^{(2)}\right)\right]
\end{aligned}
$$


Using Eq.(B3), the expansion terms on the left side of the equation cancel and we have

$$
\begin{aligned}
N_{u} \Upsilon^{a} \Upsilon^{b} \sigma_{a b} & =N_{\Upsilon} B \sin \alpha \cos \alpha\left[\Upsilon^{a}\left(\dot{U}_{a}^{(1)}-\dot{U}_{a}^{(2)}\right)+N_{\Upsilon} \frac{\theta_{(2)} \cos \alpha+\theta_{(1)} B \sin \alpha}{3}\right. \\
& \left.+\Upsilon^{a} \Upsilon^{b}\left(\cos \alpha \sigma_{a b}^{(1)}+B \sin \alpha \sigma_{a b}^{(2)}\right)\right]
\end{aligned}
$$

The shear has zero trace and since $\sigma_{a b} X^{a} X^{b}$ and $\sigma_{a b} Z^{a} Z^{a}=0$, for shear-free components this requires $\Upsilon^{a} \Upsilon^{b} \sigma_{a b}=0$. A composite shear-free condition is

$$
\Upsilon^{a}\left(\dot{U}_{a}^{(2)}-\dot{U}_{a}^{(1)}\right)=N_{\Upsilon} \frac{\theta_{2} \cos \alpha+\theta_{1} B \sin \alpha}{3}
$$

\section{Vorticity}

The vorticity follows from the antisymmetrized Eq.(B7). The $\Upsilon^{a} X^{b}$ contraction is

$$
\Upsilon^{a} X^{b} \omega_{a b}=\left[\frac{B \sin \alpha \cos \alpha N_{\Upsilon}}{2 N_{u}}\right] X^{b}\left(\dot{U}_{b}^{(2)}-\dot{U}_{b}^{(1)}\right)+\frac{\Upsilon^{a} X^{b}}{N_{u}}\left(\cos \alpha \omega_{a b}^{(1)}+B \sin \alpha \omega_{a b}^{(2)}\right)
$$

and similarly

$$
\begin{aligned}
\Upsilon^{a} Z^{b} \omega_{a b} & =\left[\frac{B \sin \alpha \cos \alpha N_{\Upsilon}}{2 N_{u}}\right] Z^{b}\left(\dot{U}_{b}^{(2)}-\dot{U}_{b}^{(1)}\right)+\frac{\Upsilon^{a} Z^{b}}{N_{u}}\left(\cos \alpha \omega_{a b}^{(1)}+B \sin \alpha \omega_{a b}^{(2)}\right) \\
Z^{a} X^{b} \omega_{a b} & =\frac{Z^{a} X^{b}}{N_{u}}\left(\cos \alpha \omega_{a b}^{(1)}+B \sin \alpha \omega_{a b}^{(2)}\right)
\end{aligned}
$$

\section{Appendix C: Fluid Acceleration and Expansion for shear-free components}

The component fluids that will combine to create a shear-free composite have strong restrictions on their accelerations and expansions. The shear of the composite fluid is the most important of the composite parameters as its form will set the component conditions. From Eqs.(B17,B19), for shear-free components, the composite shear tensor components are

$$
\begin{aligned}
X^{a} X^{b} \sigma_{a b} & =Z^{a} Z^{b} \sigma_{a b}=X^{a} Z^{b} \sigma_{a b}=0 \\
X^{a} \Upsilon^{b} \sigma_{a b} & =\left[\frac{B \sin \alpha \cos \alpha N_{\Upsilon}}{2 N_{u}}\right] X^{a}\left(\dot{U}_{a}^{(1)}-\dot{U}_{a}^{(2)}\right) \\
Z^{a} \Upsilon^{b} \sigma_{a b} & =\left[\frac{B \sin \alpha \cos \alpha N_{\Upsilon}}{2 N_{u}}\right] Z^{a}\left(\dot{U}_{a}^{(1)}-\dot{U}_{a}^{(2)}\right) \\
\Upsilon^{a} \Upsilon^{b} \sigma_{a b} & =\frac{N_{\Upsilon}}{N_{u}} B \sin \alpha \cos \alpha\left[\Upsilon^{a}\left(\dot{U}_{a}^{(1)}-\dot{U}_{a}^{(2)}\right)+N_{\Upsilon}\left(\frac{\theta_{(2)} \cos \alpha+\theta_{(1)} B \sin \alpha}{3}\right)\right]
\end{aligned}
$$


An inspection of the composite shear establishes two conditions for the composite fluid to be shear-free.

$$
\begin{aligned}
& X^{a}\left(\dot{U}_{a}^{(1)}-\dot{U}_{a}^{(2)}\right)=Z^{a}\left(\dot{U}_{a}^{(1)}-\dot{U}_{a}^{(2)}\right)=0 \\
& \Upsilon^{a}\left(\dot{U}_{a}^{(2)}-\dot{U}_{a}^{(1)}\right)=N_{\Upsilon}\left(\frac{\theta_{(2)} \cos \alpha+\theta_{(1)} B \sin \alpha}{3}\right)
\end{aligned}
$$

For aligned fluids, $U_{a}^{(1)}=U_{a}^{(2)}$, these are identities with equal accelerations and $\alpha=\alpha_{0}$. Shear-free aligned fluids will produce a shear-free composite but it is isotropic and has no heat flow. For unaligned fluids, two possible conditions are equal or unequal component accelerations. The fluid overlap, $\digamma$, is assumed constant and establishes a relation between the component accelerations. From Appendix A we have

$$
\begin{aligned}
\dot{U}_{a}^{(1)} U^{(2) a} & =\left(\digamma \frac{\theta_{(1)}}{3}+\frac{\theta_{(2)}}{3}\right) \\
\dot{U}_{a}^{(2)} U^{(1) a} & =\left(\frac{\theta_{(1)}}{3}+\digamma \frac{\theta_{(2)}}{3}\right)
\end{aligned}
$$

Equal component acceleration requires $\theta_{1} \digamma=-\theta_{2}$ and $\theta_{2} \digamma=-\theta_{1}$. This implies the component fluids are aligned, $\digamma^{2}=1$. Two unaligned fluids with equal accelerations will not produce a shear-free composite. The fluid accelerations should have non-canceling components along the direction of anisotropy.

[1] P.S. Letelier, Phys. Rev. D 22, 807 (1980). Anisotropic fluids with two perfect-fluid components

[2] L. Herrera and N.O. Santos, Phys. Rep. 286, 53 (1997). Local Anisotropy in self-gravitation systems.

[3] N. Andersson and G.L. Comer, "Relativistic Fluid Dynamics, Physics for Many Different Scales", Living Rev. Relativity 10, 1 (2007)

[4] C.S. Lopez-Monsalvo and N. Andersson, Proc. Roy. Soc. A 467, 738 (2011). Thermal dynamics in general relativity.

[5] N. Andersson and G.L. Comer, Proc. Roy. Soc. A 466, 1373 (2010). Variational multi-fluid dynamics and causal heat condutivity

[6] S.G. Ghosh, Int. J. Mod. Phys. A 23, 4245 (2008). Non-spherical collapse of a two fluid star.

[7] L.M. Lin, N. Andersson and G.L. Comer, Phys. Rev. D 78, 083008 (2008). Oscillations of General Relativistic Multi-fluid/Multi-layer Compact Stars 
[8] J. Hoyos, A. Reisenegger, J.A. Valdivia, Astron. and Astrophys. 487, 789 (2008). Magnetic Field Evolution in Neutron Stars: One-Dimensional Multi-Fluid Model

[9] N. Chamel, Mon. Not. Roy. Astr. Soc. 388, 737 (2008). Two-fluid models of superfluid neutron star cores

[10] W. Zimdahl and D. Pavon, Gen. Rel. Gravit. 33, 791 (2001). Cosmological two-fluid dynamics.

[11] A. Gromov, Yu. Baryshev and P. Teerikorpi, Astron. and Astrophys. 415, 813 (2004). Twofluid matter-quintessence FLRW models: energy transfer and the equation of state of the universe.

[12] N. Pinto-Neto, E.E. Santini and F.T. Falciano, Phys. Lett. A 344, 131 (2005). Quantization of Friedmann cosmological models with two fluids: dust plus radiation.

[13] R.M. Gallis and N.E. Frankel, J. Math. Phys. 47, 062505 (2006). Two-Component Cosmological Fluids with Gravitational Instabilities

[14] N. Cruz, S. Lepe and F. Pena, Phys. Lett. B 633, 338 (2008). Dark energy interacting with two fluids

[15] I. Aref'eva, P.H. Frampton and S. Matsusaki, Proc. Steklov Inst. Math. 265, 59 (2009). Multifluid Models for Cyclic Cosmology,

[16] T. Harko and F.S.N. Lobo, Phys. Rev. D 83, 124051 (2011). Two-fluid dark matter models

[17] J.P. Krisch and E.N. Glass, J. Math. Phys. 52, 102503 (2011). Velocity and Heat Flow in a Composite Two Fluid System

[18] J. Carminati and E. Zakhary, Class. Quantum Grav. 16, 3221 (1999). Classsification and compactibility of energy-momentum tensors

[19] M. Goliath and U.S. Nilsson, J. Math. Phys. 41, 6906 (2000). Isotropization of two-component fluids.

[20] M. Cissoko, Phys. Rev. D 63, 083516 (2001). Wave fronts in a mixture of two relativistic perfect fluids flowing with two distinct four-velocities

[21] R.D. Zarate and H. Quevedo, Class. Quantum Grav. 21, 197 (2004). Thermodynamic scheme of inhomogeneous perfect fluid mixtures.

[22] L. Herrera, N.O. Santos and A. Wang, Phys. Rev. D 78, 084026 (2008). Shearing Expansion Free Spherical Anisotropic Fluid Evolution

[23] R.J. Wiltshire, Gen. Rel. Gravit. 44. 941 (2012). A Rotating Three component perfect fluid source and its junction with empty space-time. 
[24] N.A. Koshelev, JCAP 1104:021(2011), arXiv:astro-ph/1011.0569 Non-adiabatic perturbations in multi-component perfect fluids.

[25] V. Marra and M. Paakkonen, JCAP 01(2012)025 arXiv:gr-qc/1105.6099 Exact sphericallysymmetric inhomogeneous model with $n$ perfect fluids

[26] P.K.S. Dunsby, Class. Quantum Grav. 8, 1785 (1991). Gauge invariant perturabations in multi-component fluid cosmologies

[27] O. Bergmann, Phys. Lett A 82, 383 (1981). A Cosmological Solution Of The Einstein Equations With Heat Flow

[28] A.K. Sanyal and D. Ray, J. Math. Phys. 25, 1975 (1984). Cosmological solutions of the Einstein equation with heat flow

[29] Y. Deng, Gen. Rel. Gravit. 21, 503 (1989). Solutions of the Einstein Equation with Heat Flow.

[30] G.F.R. Ellis, Gen. Rel. Gravit. 43, 3253 (2011). Shear free solutions in General Relativity Theory.

[31] C.B. Collins, Can. J. Phys. 64, 191 (1986). Shear-free fluids in general relativity

[32] C.F. Sopuerta, Class. Quantum Grav. 15, 1043 (1998) Covariant study of a conjecture on shear-free barotropic perfect fluids

[33] N. Van den Bergh, Class. Quantum Grav. 16, 117 (1999). The shear-free perfect fluid conjecture

[34] N. Van den Bergh, J. Carminati, and H. Karimian, Class. Quantum Grav. 24, 3735 (2007). Shear-free perfect fluids with solenoidal magnetic curvature and a gamma-law equation of state

[35] J. Carminati, H.R. Karimian, N. Van den Bergh, and K.T. Vu, Class. Quantum Grav. 26, 195002 (2009). Shear-free perfect fluids with a solenoidal magnetic curvature

[36] A. Krasinski, Inhomogeneous Cosmological Models, (Cambridge University Press, 1997, Ch. $4)$.

[37] H. Strobel, Wiss. Z. Friedrich Schiller Univ. Jena, Math. Naturw. Reihe 17,195 (1968) and 21, 111 (1972). This is reviewed by A. Krasinski [36]

[38] R.A. Sussman, Class. Quantum Grav. 10, 2675 (1993). New solutions for heat conducting fluids with a normal shear-free flow

[39] G.L. Comer, P. Peter, and N. Andersson, Phys. Rev. D 85, 103006 (2012). Multi-Fluid Cosmology: An illustration of fundamental principles

[40] L. Samuelsson, C.S. Lopez-Monsalvo, N. Andersson, and G.L. Comer, Gen. Rel. Gravit. 42, 
413 (2010). Relativistic two-stream instability.

[41] S. Ragusa and M. Bailyn, Gen. Rel. Gravit. 27, 163 (1995). The center of Trace in Spinning Particles in General Relativity

[42] O. Costa, J, Natário, and M. Zilhão, Mathisson's helical motions demystified AIP Conf. Proc. 1458, 367-370 (2011).

[43] L. Filipe, O. Costa, C.A.R. Herdeiro, J. Natário, and M. Zilhão, Phys. Rev. D 85, 024001 (2012). Mathisson's helical motions for a spinning particle - are they unphysical?

[44] B.F. Schutz, Phys. Rev. D 2, 2762 (1970). Perfect Fluids in General Relativity: Velocity Potentials and a Variational Principle.

[45] D. Van Dantzig, Physica VI(8), 673 (1939). On the Phenomenological Thermodynamics of Moving Matter. 